\title{
Biophysics and Thermodynamics: The Scientific Building Blocks of Bio-inspired Drug Delivery Nano Systems
}

\author{
Costas Demetzos ${ }^{1,2}$
}

Received 9 March 2015; accepted 7 April 2015; published online 22 April 2015

\begin{abstract}
Biophysics and thermodynamics are considered as the scientific milestones for investigating the properties of materials. The relationship between the changes of temperature with the biophysical variables of biomaterials is important in the process of the development of drug delivery systems. Biophysics is a challenge sector of physics and should be used complementary with the biochemistry in order to discover new and promising technological platforms (i.e., drug delivery systems) and to disclose the 'silence functionality' of bio-inspired biological and artificial membranes. Thermal analysis and biophysical approaches in pharmaceuticals present reliable and versatile tools for their characterization and for the successful development of pharmaceutical products. The metastable phases of self-assembled nanostructures such as liposomes should be taken into consideration because they represent the thermal events can affect the functionality of advanced drug delivery nano systems. In conclusion, biophysics and thermodynamics are characterized as the building blocks for design and development of bio-inspired drug delivery systems.
\end{abstract}

KEY WORDS: biophysics; drug delivery nano systems; pharmaceutics; thermal analysis; thermodynamics.

\section{INTRODUCTION TO PHARMACEUTICAL NANOTECHNOLOGY}

Fabricated nano-sized devices or drug carriers, often called nanocarriers or nano-vehicles, provide various advantages for effective drug delivery. Nanocarriers can carry poorly soluble, unstable, or systemically toxic drugs with extended blood half-lives and reduced side effects (1). Pharmaceutical nanotechnology, which is an advantageous approach for developing innovative drugs and drug carriers, can provide challenges for producing bio-inspired and self-assembled nanostructures that can be termed as advanced drug delivery nano systems (aDDnSs). Advanced drug delivery nano systems can be characterized as mixed nano systems due to the combination of different in nature bionanomaterials and are used for biomimetic delivery (2). The advanced drug delivery nano systems are characterized as modulatory controlled release nano systems and were classified in hybrid and chimeric (i.e., same and different in nature biomaterials) (3-5). Additionally, nanosciences and nanotechnology compromise new approaches to the research field of pharmaceutics. These fields have the ability of understanding new interfacial

Prof. Costas Demetzos is elected member of Executive Committee in European Federation of Pharmaceutical Sciences (EUFEPS) (Network Coordinator)

\footnotetext{
${ }^{1}$ Department of Pharmaceutical Technology, Faculty of Pharmacy, National and Kapodistrian University of Athens, Panepistimioupolis Zografou 15771, Athens, Greece.

${ }^{2}$ To whom correspondence should be addressed. (e-mail: demetzos@pharm.uoa.gr)
}

phenomena and show new properties of these materials. The real challenge is that the nanostructures that are created do not conform to the classic laws of physics due to their size but are too big to follow the principles of quantum mechanics. These systems belong to a scale that is called mesoscale. As a result, there is the appearance of a new scientific field, despite of the scientific approaches of the past centuries in the dimensions of macro- and microworld. This way, the science of mesoscale and mesoworld is the new challenge, where the nano dimension is the ruling size for the creation of structures and systems with new properties. The interfacial phenomena in this dimension are the ones ruling due to the large overall surface of the nano system and due to the fact that most of the atoms of these materials are on their surface. The creation of nanostructures in molecular level aiming the self-assembly that would function in molecular level but also in tissue regeneration are prerequisites for nanotechnology in the health sciences.

Biological systems contact biomaterials, e.g., for pharmaceutical applications like controlled drug release systems produced by biomaterials (monoclonic antibodies, macromolecules) in order to target tissues, etc. We can highlight that biomaterials and other biological components interact with biologic system surfaces developing interfacial interactions. Biomaterial adsorption into cell affects their biological functionality. Pharmaceutical products in colloidal state can affect various systems functions possibly improving them in human favor. The pharmaceutical industry tries to develop high-quality colloidal biomaterials, emphasizing in controlling their properties so when in the appropriate environment, they will influence it to the desired direction. We highlight that colloidals are the systems that 
the dispersed particles are in sizes between a few nanometers to a few thousand nanometers.

\section{BIOPHYSICS APPLIED TO DRUG DELIVERY SYSTEMS}

Biophysics is a valuable tool for better understanding the various self-assembled organization levels of life, from biomolecules to ecosystems. However, biophysics is a challengeapplied sector of physics and is used complementary to chemistry, biosciences, applied mathematics, and micro- and/or nano-engineering, as well as nanotechnology. In order to disclose new and promising technological platforms and to reveal the "silence functionality" of bio-inspired drug delivery nano systems, the role of interfacial phenomena can be related to the membranes' biophysical behavior as well as with their thermotropic changes (i.e., flip-flop and metastable phases). As an example of the biophysical approach in the development of drug delivery nano systems, we can refer to the phase transitions of the liquid crystalline phase that are present in the cells and correlate with their functionality, stability, and effectiveness. Therefore, the development of drug delivery nano systems, like liposomes, dendrimers, nanoemulsions, polymers, etc., that could transfer bioactive molecules into the cells of damaged tissues simulate the function of the living cells. However, the silence functionality is encoded into metastable phases and combined both the biophysical and the thermotropical behavior of an advanced drug delivery nano system. The metastable phases are investigated by model lipid membranes with thermo-analytical techniques. It should be noted that the most of them are kinetically trapped in a stable macroscopic fingerprint. These metastable phases act as modulators, promoters, or retarders of physical phenomena that are macroscopically observed. Simulating artificial membranes composed of building blocks as those that occur in living cells (i.e., phospholipids), we can have a complete picture of the biophysical behavior of drug delivery systems by using the laws and the principles of thermodynamics and biophysics. Phenomena such as aggregation process, fusion of particles, and creations of biophysical 'islands' as domains into artificial bilayers affect their organization, their distribution of size, and their elucidation of the shape and morphology and could be considered as predominant segments in order to rationally design, develop, and evaluate technological dosage forms and new nanoplatforms for drug delivery. For these senses, it is important to take into consideration that the principles of the cell behavior, such as stability, diversity, and functionality, should be transferred in the level of designing and developing drug delivery nano systems and consequently innovative medicinal and health products. This bio-inspired approach for design and develop nanoparticulate drug delivery systems is a scientific trend due to several similarities of the building blocks (i.e., bionanomaterials) of living cells and advanced drug delivery nano systems (6-10).

\section{THERMODYNAMICS: THE PHYSICAL BEHAVIOR OF ADVANCED DRUG DELIVERY NANO SYSTEMS}

Thermodynamics, historically translates the natural behavior by describing physically the diversity and the polyvalence in the evolution process of living organisms. The balance between the adaptation processes of biological objects was internally governed by the thermodynamic equilibrium processes in each state of the material transitions. The metastable phases play a key role on their functionality by regulating the "switching on and off" of the processes (i.e., the transmembrane communication). According to Lasic (1990), the metastable phases of liposomes (model of lipid membranes) could be characterized as "kinetically trapped" (11). Additionally, according to our recent published papers, there is a strong relationship between the metastable phase and the mechanistic explanation of the biophysical behavior of advanced drug delivery nano systems. Namely, the metastable phases of mixed liposomal membranes control the release of the encapsulated drug (9). Furthermore, according to the proposed balance of the physicochemical/thermodynamic characteristics of nano systems, there is a strong interplay between their curvature (morphology) and their thermal behavior because the observed metastable phases are entropic traps that govern their functionality $(11,12)$. These metastable phases must be probed during the pre-formulation studies in order to develop innovative nanocarriers with complete knowledge of their characteristics and produce safe and effective medicines. However, the physicochemical/thermodynamical balance and the transitions from one phase of the matter to another, by lowering the change of the free energy $(\Delta \mathrm{G})$ of the system, are the driving forces for maintaining the quality of the evolution process in nature, through time. In other words, we should take advantage of the existence of the metastable phases in order to produce systems that mimic the excellence of the physical processes.

Thermal analysis is considered as one of the most popular techniques in material sciences and engineering and is a highly sensitive technique to study the thermotropic properties of many different macromolecules. Thermal analysis has been applied to the pharmaceutical field for studying excipients, biomaterials, and drugs. Applications of this technique to lipid-based systems such as artificial biological membranes include the measurement of thermodynamic parameters and a detailed characterization of thermotropic and phase transition behavior (3).

Furthermore, thermal analysis casts light in a total new scientific perspective by treating drugs as biomaterials and not as plain materials. In the pharmaceutical sciences, only a handful of techniques are commonly employed, but the information gained and phenomena, like aggregation, that can be explored are countless. As formulations become more and more complex and characterizing them becomes more difficult, manufacturers have done an excellent work in keeping pace with more precise and sensitive yet more durable instruments. Especially, advanced drug delivery technologies focus on the connection between physicochemical characteristics (polymorphism, fluidity, surface charge, thermotropic behavior of lipidic membranes, etc.) of membranes with the alteration of pharmacokinetics and bioavailability of drugs. The biophysical and the thermodynamic approaches for the design and development of nano systems and advanced drug delivery platforms can be correlated with their phase transitions, i.e., from gel $\left(\mathrm{L}_{\beta}\right)$ to liquid crystalline phase $\left(\mathrm{L}_{\alpha}\right)$, possible through metastable phases (ripple phase, $\mathrm{P}_{\beta}$ ) depending on the chemical and physicochemical properties of the building blocks of the system. Moreover, these metastable phase transitions are 
considered as 'thermodynamical vehicles' that could be correlated with the functionality of the nano system and the effectiveness of the nanoparticulate medicine (i.e., release and kinetics properties). In our point of view, the metastable phases are considered as key elements for studying the behavior of bio-substances (i.e., macromolecules or bioactive compounds, respectively) incorporated into bio- and artificial membranes.

As an example of the biophysical approach in the development of nano systems, we can refer to the phase transitions of the liquid crystalline phase that is present in the cells and correlate with their functionality, stability, and effectiveness. Therefore, there could be a development of nano systems, like liposomes, dendrimers, nanoemulsions, polymers etc., that could transfer bioactive molecules into the cells of damaged tissues and release them according to the decoding of the hidden to them-according to the science of nanotechnology-silence functionality, simulating the function of the living cells.

The liquid crystal phase, the study of the nano system thermodynamics as it is quantified from the physics laws, the study of their thermotropic behavior as it is known from the functionality of nano systems in correlation to their physicochemical characteristics-for example, the study and the evaluation of size and distribution of sizes in colloidal dispersions in reference to the biocolloidal dispersions of living organisms - are very important physical approaches in pharmaceutics and comply with the science of biophysics. From all the above, we conclude that we can build nanostructures and nano systems, study them, and evaluate them making nanotechnological products that can be used in disease diagnosing, damaged tissue imaging, and their cure.

\section{THE INTERPLAY BETWEEN THE METASTABLE PHASES AND THE BIOPHYSICAL BEHAVIOR OF ADVANCED DRUG DELIVERY NANO SYSTEMS}

As mentioned above, the term "metastable phase" is given to a thermodynamic instant nonequilibrium state of a substance or a state of matter (phase) whose properties alter reversibly over time. In the real systems, we can state that there are metastable phases present kinetically during the natural processes. These metastable phases are also present to the cellular membranes.

It is well documented in the literature that metastable phases can act as modulators of the release of the incorporated bioactive molecule, due to the structural rearrangement of the artificial lipidic bilayers such as liposomes, affecting their behavior and consequently their functionality, as differential scanning calorimetry (DSC) experiments indicated (9). The biomaterials produce metastable phases working like a 'zipper' that are correlated to the biophysical properties of biomembranes introducing the 'tunneling' effects. The tunneling effects help scientists to understand the biophysical functionality of bio-channels and also facilitate the design and development of innovative nanostructures. These innovative nanostructures transport and deliver the bioactive molecules to the right target, resulting in the reduction of side effects and the efficacy of the final medicinal product. Moreover, the interfacial phenomena that are played sufficient role in membranes' survival and functionality were almost impossible to discover and to characterized and measure all their physicochemical parameters. In order to simplify these complicated systems, simple building blocks of biomembranes such as lipids, phospholipids, cholesterol, etc. are recognized as the main biomaterials that are be able to behave in thermodynamically and biophysical point of view as the living cell membranes.

\section{The Self-Assembly Behavior of Bionanomaterials}

The changes of the enthalpy $(\Delta \mathrm{H})$ as a function of the phase transitions have been already studied by DSC, which is a relatively inexpensive technique that allows the study of the thermotropic properties of membranes $(3,4)$. It is well documented from the literature and from the results and the data published that a huge number of different metastable mesophases were recognized and characterized by thermodynamics, by using lipid bilayers as models of cell membranes. In an effort to reproduce such systems, scientists discovered the kind and chemical properties of biomaterials that are able to organize in such a way that offer the self-assembly profile as the living cell membranes and could be used as artificial platforms (i.e., liposomes) for producing drug delivery systems $(8,9)$. Taking into account the thermodynamics of the cell membranes composed of a huge number of different innature biomaterials, there is a weakness to reproduce identically the cell membranes.

It is also well known that formation of unique structures of biological macromolecules, such as proteins and their specific complexes, is, in principle, reversible, and the reactions are thermodynamically driven. Therefore, thermodynamic investigations of these processes are of high priority. To achieve this goal, direct measurements of the heat effects associated with these intra- and intermacromolecular processes are required to facilitate supersensitive calorimetric techniques such as DSC, which is considered one of the most frequently used techniques to determine thermal stability of proteins and to measure the thermodynamic parameters of thermal protein unfolding. The application of calorimetric methods to study the thermodynamics of nucleic acid-folding transitions has gradually been improved in recent years. These improvements lead to the production of high-precision microcalorimeters (13-15).

The principle phases formed by soft condensed matter are due to interplay between positional, orientational, and conformational disorder. There are a wide variety of different mesophases that can be further refined within the broad category of liquid crystalline materials, i.e., materials with orientational and conformational ordering. Furthermore, many biological molecules are chiral (e.g., DNA is normally left handed), and their mesophase structure reflects the chiral interaction between subunits.

Thermal analysis techniques reveal the presence of liquid crystalline phase transitions in a material through the detection of the associated enthalpy changes. Diffusion is the process by which molecules jiggle around over small distances due to thermal collisions with their neighbors (Brownian motion), and equivalently, diffusion can be used to explain how macroscopic concentration gradients in materials evolve with time. Biological complexes are often extremely complicated, and it was an important advance when many were found to self- 
assemble on a mesoscopic level from their 'raw ingredients'. Bilayers are easily created synthetically from a range of lipid molecules and spontaneously arrange themselves into vesicles. Naturally occurring cell membranes follow more complicated schemes of construction (their structures include intramembrane proteins and scaffolding). However, the underlying scheme of amphiphilic self-assembly is still thought to hold. A distinction is made between examples of aggregating self-assembly (e.g., micellisation of lipids) and non-aggregating self-assembly (e.g., folding of globular proteins). Aggregating self-assembly has some conceptually sophisticated universal thermodynamic features (e.g., a critical micelle concentration) which are considered in detail in this chapter. Non-aggregating self-assembly usually describes the behavior of a system that moves between some hidden free energy minima $(16,17)$.

Other more general examples of self-assembly exist in soft-condensed matter physics, such as the morphologies produced in the phase separation of liquids, liquid-crystals, polymers and block copolymers, liposomes, and mixed systems $(8,9)$. All of these have analogues in molecular biophysics.

It is well known that the second law of thermodynamic deals with the change of entropy $(\triangle S)$ and fights nature integrity and consequently humans' infinity. Biological systems such as cells, cells' organelles, viruses, as well as biomaterials essential for human beings' evolution, such as DNA, RNA, and the consequences from their functionality such as proteins, peptides, hormone, etc., are affected by the law of entropy which proceed them to the end of the lifeline (i.e., deadline). The equilibrium states referred as metastable mesophases of the mater and the bounders between different phases of matter are characterized as equilibrium lines on which the evolution process of the system to a higher quality and functionality is realized. The thermodynamics in such systems was recognized as essential and provide new insights to design and to produce effective new dosage forms which reflect to the applied pharmaceutics $(18,19)$.

The basic elements provided by the natures' evolution were presented as the toolkits in a scientific toolbox that is attractive for studying new and innovative bio-inspired platforms useful for the human health care. Pharmaceutical nanotechnology does not aspire to be so precise keeping its efforts to the efficiently design and develop of nanostructures based on technological platforms. These nanostructured systems could be correlated to the living organisms due to their selfassembly to their hierarchical structural organization, as well as to their biocompatibility and biodegradability characteristics. However, drug delivery systems can be considered as new therapeutic outcomes in nanomedicine that could be able to deliver bioactive compounds to specific tissues and can improve their PK/PD (pharmacokinetics/pharmacodynamics) behavior and affect their total bioavailability.

\section{THE MARKET CHALLENGES AND THE REGULATORY PERSPECTIVES}

We have to remark that thermodynamics and biophysics are efficiently contributed in the evaluation process concerning the properties of drug delivery systems. Studies that determine the interactions between biomaterials as well as their interactions with the biological media can be achieved by using thermal analysis techniques. These scientific blocks of bio-inspired drug delivery systems play a key role in the "cycle of innovation" of nanotechnology-based products. The drugdevice combinations are of paramount importance in order to develop nanoparticulate medicines.

Scientists have used these techniques in order to physicochemically characterize nano systems and nanoparticles like liposomes, dendrimers, polymers, and nanotechnological drugs that are in the market (i.e., liposomal amphotericin and doxorubicin). More in particular, these characterization techniques refer to the study of nanoparticles properties, i.e., measurement of the width of the liposomal lipid membrane, the ability of allowing biomolecules to penetrate their membrane, the bioactive molecules loading ability not only for therapeutic purposes but also for tissue diagnosis and imaging. Also, the nano system stability in biological fluids over time is a part of studying and characterizing these properties according to the methods mentioned before. The nanoparticles physicochemical characterization is a process that includes high technological studies and requires great expertise in result evaluation. These techniques relate, for example, to nanoparticle size count and size distribution, to $\zeta$-potential measure, to shape/morphology studies, to bioactive molecule enclosure capacity, and to physicochemical characterization of a bioactive molecule nano system, etc.

Additionally, thermodynamics and biophysics contribute significantly to innovative therapeutic approaches. According to Professor Meindert Danhof Fundamental Sciences contribute to the Systems Therapeutics, which are based to System Biology and the analysis of biological network. The cycle of innovation and the "Systems Therapeutics" are in line in order to produce new safe medicines with added value for public health. The shift to the fundamental sciences helps to translational, formulation, and manufacturing sciences and creates the scientific basis for regulatory sciences. For these reasons, biophysics and thermodynamics could create the science-based platform for Systems Therapeutics

Recently, from our research group (there are several publications concerning the thermotropic behavior of nanoparticles), a comment on "Guideline on process validation for the manufacture of biotechnology-derived active substances and data to be provided in the regulatory submission $>$ ' (EMA/ CHMP/BWP/187338/2014)" was submitted to European Medicines Agency (EMA). This comment included the adoption of biophysical and thermodynamic characteristics in order to completely evaluate biotechnology-derived active substances. By this submitted comment is bridged the gap between the fundamental sciences (i.e., thermodynamics and biophysics) and translational and manufacturing sciences, as required from the regulatory approaches.

\section{CONCLUSIONS}

In order to conclude the above trends in advanced drug delivery systems, we can adapt the well known term "bioinspired" in order to promote the biomimetic concept as the mirror of the "thermodynamic vesicle" for producing innovative drug delivery nano systems based on their evaluation by using elements/building blocks of from thermodynamics and biophysics. This approach is in line with the contribution of fundamental sciences to innovative therapeutic approaches, as 
the "cycle of innovation" which is the map of the EU's regulation of new health technologies demands.

\section{REFERENCES}

1. Crommelin DJ, Florence AT. Towards more effective advanced drug delivery systems. Int J Pharm. 2013;454:496-511.

2. Balmert SC, Little SR. Biomimetic delivery with micro- and nanoparticles. Adv Mater. 2012;24:3757-78.

3. Demetzos C. Differential scanning calorimetry (DSC): a tool to study the thermal behavior of lipid bilayers and liposomal stability. J Liposome Res. 2008;18:159-73.

4. Demetzos C. Special chapter on current advancement of thermal analysis with special focus on bio- and pharmaceutical researchers from the Mediterranians. J Therm Anal Calorim. 2014;116:1-3.

5. Gardikis K, Tsimplouli C, Dimas K, Micha-Skretta M, Demetzos C. New chimeric advanced drug delivery nanosystems (ChiaDDnSs) as doxorubicin carriers. Int J Pharm. 2010;402:231-7.

6. Gardikis K, Hatziantoniou S, Signorelli M, Pusceddu M, MichaScretta M, Schiraldi A, et al. Thermodynamics and structural characterization of liposomal locked-in dendrimers as drug carriers. Colloids Surf B: Biointerfaces. 2010;81:11-9.

7. Gardikis K, Fessas D, Signorelli M, Dimas K, Tsimplouli C, Ionov M, et al. A new chimeric drug delivery nano system (chi-aDDnS) composed of PAMAM G 3.5 dendrimer and liposomes as doxorubicin's carrier. In vitro pharmacological studies. J Nanosci Nanotechnol. 2011;5:3764-72.

8. Pippa N, Gardikis K, Pispas S, Demetzos C. The physicochemical/ thermodynamic balance of advanced drug liposomal delivery systems. J Ther Anal Calorim. 2014:116:99-105.

9. Pippa N, Pispas S, Demetzos C. The metastable phases as modulators of biophysical behavior of liposomal membranes. The role of the biomolecular sculpture of the polymeric guest. J Ther Anal Calorim. 2015; In press.

10. Le Meins J-F, Schatz C, Lecommandoux S, Sandre O. Hydrid polymer/lipid vesicles: state of the art and future perspectives. Mat Today. 2013;16:397-402.

11. Lasic DD. On the thermodynamic stability of liposomes. J Colloid Interface Sci. 1990;1:302-4.

12. Lim SK, de Hoog H-P, Parikh AN, Nallani M, Liedberg B. Hybrid nanoscale phospholipid/block copolymer vesicles. Polymers. 2013;5:1102-14.

13. Takano K, Yamagata Y, Fujii S, Yutani K. Contribution of the hydrophobic effect to the stability of human lysozyme: calorimetric studies and X-ray structural analyses of the nine valine alanine mutants. Biochemistry. 1997;36:688-98. 64.

14. Takano K, Yamagata Y, Kubota M, Funahashi J, Fujii S, Yutani $\mathrm{K}$. Contribution of hydrogen bonds to the conformational stability of human lysozyme: calorimetry and X-ray analysis of six Ser Ala mutants. Biochemistry. 1999;38:6623-9.

15. Vogl T, Jatzke C, Hinz HJ, Benz J, Huber R. Thermodynamic stability of annexin VE17G: equilibrium parameters from an irreversible unfolding reaction. Biochemistry. 1997;36: 1657-68.

16. Gill P, Moghadam TT, Ranjbar B. Differential scanning calorimetry techniques: applications in biology and nanoscience. $\mathrm{J}$ Biomol Tech. 2010;21(4):167-93.

17. Johnson CM. Differential scanning calorimetry as a tool for protein folding and stability. Arch Biochem Biophys. 2013;531(1-2):100-9.

18. Bruylants G, Wouters J, Michaux C. Differential scanning calorimetry in life science: thermodynamics, stability, molecular recognition and application in drug design. Curr Med Chem. 2005;12(17):2011-20.

19. Ford JL, Mann TE. Fast-Scan DSC and its role in pharmaceutical physical form characterization and selection. Adv Drug Deliv Rev. 2012;64(5):422-30. 